\title{
Video Article \\ Analysis of Mitochondrial Respiratory Chain Complexes in Cultured Human Cells using Blue Native Polyacrylamide Gel Electrophoresis and Immunoblotting
}

\author{
Svetlana Konovalova ${ }^{1}$ \\ ${ }^{1}$ Research Programs Unit, Molecular Neurology, University of Helsinki \\ Correspondence to: Svetlana Konovalova at svetlana.konovalova@helsinki.fi
}

URL: https://www.jove.com/video/59269

DOI: doi:10.3791/59269

Keywords: Biology, Issue 144, Mitochondria, OXPHOS complexes, blue native PAGE, electrophoresis, respiratory chain complexes, mitochondrial proteostasis

Date Published: 2/12/2019

Citation: Konovalova, S. Analysis of Mitochondrial Respiratory Chain Complexes in Cultured Human Cells using Blue Native Polyacrylamide Gel Electrophoresis and Immunoblotting. J. Vis. Exp. (144), e59269, doi:10.3791/59269 (2019).

\section{Abstract}

Mitochondrial respiration is performed by oxidative phosphorylation (OXPHOS) complexes within mitochondria. Internal and environmental factors can perturb the assembly and stability of OXPHOS complexes. This protocol describes the analysis of mitochondrial respiratory chain complexes by blue native polyacrylamide gel electrophoresis (BN-PAGE) in application to cultured human cells. First, mitochondria are extracted from the cells using digitonin, then using lauryl maltoside, the intact OXPHOS complexes are isolated from the mitochondrial membranes. The OXPHOS complexes are then resolved by gradient gel electrophoresis in the presence of the negatively charged dye, Coomassie blue, which prevents protein aggregation and ensures electrophoretic mobility of protein complexes towards the cathode. Finally, the OXPHOS complexes are detected by standard immunoblotting. Thus, BN-PAGE is a convenient and inexpensive technique that can be used to evaluate the assembly of entire OXPHOS complexes, in contrast to the basic SDS-PAGE allowing the study of only individual OXPHOS complex subunits.

\section{Video Link}

The video component of this article can be found at https://www.jove.com/video/59269/

\section{Introduction}

Mitochondria are multifunctional organelles playing an important role in energy production, regulation of cellular metabolism, signaling, apoptosis, aging, etc. ${ }^{1,2,3}$. The energy production in mitochondria relies on the oxidative phosphorylation function that couples respiration with ATP synthesis. In human cells, the mitochondrial oxidative phosphorylation system (OXPHOS) is composed of five complexes. Complexes I-IV create an electrochemical proton gradient in the mitochondrial intermembrane space that is used by complex V to produce ATP. Each OXPHOS complex is multimeric and, except complex II, composed of the subunits encoded by both nuclear and mitochondrial genes. Any defects in the core components of the OXPHOS complexes caused by mutations or environmental stress can perturb the assembly and functionality of the oxidative phosphorylation system. In addition, the proper assembly of functional OXPHOS complexes requires a large number of assembly factors ${ }^{4,5,6}$

Blue native polyacrylamide gel electrophoresis (BN-PAGE) is a fundamental technique enabling analysis of intact protein complexes and can be used to study the assembly of OXPHOS complexes. First, mitochondria are isolated from the cells by digitonin, which is a mild detergent that permeabilizes the plasma membrane of the cells. Then, by using lauryl maltoside, OXPHOS complexes are released from the mitochondrial membranes. By using gradient gel electrophoresis, the OXPHOS complexes are separated according to their mass. Coomassie blue G-250 (not R-250) added to the sample buffer and the blue cathode buffer dissociates detergent-labile associations but preserves individual respiratory chain complexes intact ${ }^{8}$. During the electrophoresis, the blue cathode buffer containing dye is replaced by the cathode buffer without dye, which ensures efficient transfer of OXPHOS complexes to the PVDF membrane ${ }^{8}$. To visualize OXPHOS complexes, the PVDF membrane is sequentially incubated with antibodies corresponding to the selected subunits of the five OXPHOS complexes.

The method described here with some modifications can be applied to any cultured cells. In addition, this method can be used for analysis of OXPHOS complexes in mitochondria isolated from tissue samples ${ }^{9}$. BN-PAGE requires at least 5-10 $\mu \mathrm{g}$ of mitochondria for each sample per run. Using the method described here, 500,000 cultured cells such as HEK293, SH-SY5Y or 143B cells can yield approximately $10 \mu \mathrm{g}$ of mitochondria. However, a sufficient amount of the cells for the BN analysis depends on the specific cell type.

The most common method to study mitochondrial OXPHOS proteins is SDS-PAGE and western blotting. However, SDS-PAGE allows studying only individual OXPHOS subunits and, in contrast to BN-PAGE, cannot be used to evaluate the assembly of entire OXPHOS complexes. Clear native-PAGE separates protein complexes in native conditions without the presence of negatively charged dye and has a significantly lower resolution compared to BN-PAGE. However, clear native-PAGE is milder than BN-PAGE so it can retain labile supramolecular assemblies of protein complexes such as OXPHOS supercomplexes that are dissociated under the BN-PAGE conditions ${ }^{10}$. In this protocol, the gradient gel 
is used to separate complexes; however, alternatively, non-gradient separation can be used if the relatively expensive gradient maker is not available ${ }^{11}$.

Important, the method described here allows analyzing the assembly of OXPHOS complexes, while the functionality of the complexes is not assessed. A high-resolution BN-PAGE followed by in-gel activity assay ${ }^{11}$ as well as spectrophotometric enzymatic activity assay of the mitochondrial complexes ${ }^{12}$ are efficient techniques for the functional analysis of the OXPHOS complexes. However, both of these methods do not assay the assembly of OXPHOS complexes.

Thus, BN-PAGE is the optimal method to investigate the assembly of individual OXPHOS complexes. For example, some mitochondrial disorders, such as Leber hereditary optic neuropathy (LHON), mitochondrial encephalopathy with lactic acidosis and stroke-like episodes (MELAS), are associated with altered assembly of one or more components of the OXPHOS system ${ }^{5}$. By using the BN-PAGE method described here, the molecular mechanisms of mitochondrial diseases can be studied.

\section{Protocol}

spaNOTE: This protocol is based on an associated publication by Hilander et al. ${ }^{13}$.

\section{Preparation of buffers and solutions}

NOTE: All the buffers and solutions used in the protocol are summarized in Table 1. The given volumes of the buffers are sufficient to prepare and run 10 samples. All the buffers can be stored at $+4{ }^{\circ} \mathrm{C}$ for up to one year.

1. Prepare $20 \mathrm{~mL}$ of $3 x$ gel buffer containing $1.5 \mathrm{M}$ aminocaproic acid, $150 \mathrm{mM}$ Bis-tris in distilled water ( $\mathrm{dH} \mathrm{H}_{2} \mathrm{O}$ ). Adjust $\mathrm{pH}$ to 7.0 .

2. Prepare $10 \mathrm{~mL}$ of $2 \mathrm{M}$ aminocaproic acid in $\mathrm{dH}_{2} \mathrm{O}$.

3. Prepare $1 \mathrm{~mL}$ of the mitochondrial buffer by combining the following: $0.5 \mathrm{~mL}$ of $3 x$ gel buffer, $0.5 \mathrm{~mL}$ of $2 \mathrm{M}$ aminocaproic acid and $4 \mu \mathrm{L}$ of 500 mM EDTA.

4. Prepare $1,000 \mathrm{~mL}$ of cathode buffer containing $15 \mathrm{mM}$ of Bis-tris and $50 \mathrm{mM}$ of tricine in $\mathrm{dH}_{2} \mathrm{O}$. Adjust $\mathrm{pH}$ to 7.0.

1. Prepare $200 \mathrm{~mL}$ of blue cathode buffer by adding $0.04 \mathrm{~g}$ of Coomassie blue $\mathrm{G}-250$ to $200 \mathrm{~mL}$ of cathode buffer.

5. Prepare $1,000 \mathrm{~mL}$ of anode buffer containing $50 \mathrm{mM}$ Bis-tris in $\mathrm{dH}_{2} \mathrm{O}$. Adjust $\mathrm{pH}$ to 7.0 .

6. Prepare $2.5 \mathrm{~mL}$ of sample buffer containing $750 \mathrm{mM}$ aminocaproic acid and $5 \%$ Coomassie blue $\mathrm{G}-250$ in $\mathrm{dH}_{2} \mathrm{O}$.

\section{Preparation of mitochondrial lysates}

1. Plate the cells the day before the collection. For HEK293 or 143B cells, use Dulbecco's modified Eagle's medium (DMEM) with $10 \%$ fetal bovine serum, $1 \%$ L-glutamine, $100 \mathrm{mg} / \mathrm{mL}$ penicillin and $100 \mathrm{mg} / \mathrm{mL}$ streptomycin. Grow the cells in a cell culture incubator at $+37^{\circ} \mathrm{C}$ in a $5 \% \mathrm{CO}_{2}$ humidified atmosphere. Ensure that there are at least 500,000 cells for each sample on the day of collection.

2. Gently wash the cells once with ice-cold PBS. Avoid detaching the cells from the plate. Scrape the cells and pellet them at $800 \times g$ for 10 min at $+4{ }^{\circ} \mathrm{C}$.

3. Wash the cell pellet twice with ice-cold PBS, and centrifuge as in step 2.2. Measure the protein concentration with the Bradford method using a commercial kit. Pellet the cells at $800 \times g$ for $10 \mathrm{~min}$ at $+4{ }^{\circ} \mathrm{C}$.

NOTE: After the cell collection, perform all steps at $+4{ }^{\circ} \mathrm{C}$ and do not vortex.

4. Prepare $20 \mathrm{~mL}$ of PBS with protease inhibitor by adding $200 \mu \mathrm{L}$ of $100 x$ protease inhibitor to $20 \mathrm{~mL}$ of PBS. Keep on ice. Resuspend the cells in PBS with protease inhibitor to a final protein concentration of $5 \mathrm{mg} / \mathrm{mL}$.

1. To calculate the volume of PBS with protease inhibitor needed to resuspend the cells at $5 \mathrm{mg} / \mathrm{mL}$, calculate the protein amount in each sample. For this calculation, use the protein concentration measured in step 2.3 and the volume of PBS used to resuspend the cells after washing in step 2.3 .

5. Prepare $1 \mathrm{~mL}$ of $3.3 \mathrm{mM}$ digitonin in PBS with protease inhibitor. Add $3.3 \mathrm{mM}$ digitonin to a final concentration of $1.65 \mathrm{mM}$. Mix well and incubate on ice for $5 \mathrm{~min}$.

1. To prepare $1 \mathrm{~mL}$ of $3.3 \mathrm{mM}$ digitonin in PBS with protease inhibitor, dissolve $4 \mathrm{mg}$ of digitonin in $1 \mathrm{~mL}$ of PBS at $100{ }^{\circ} \mathrm{C}$ until no precipitate is visible and cool on ice immediately. Add $10 \mu \mathrm{L}$ of $100 x$ protease inhibitor to $1 \mathrm{~mL}$ of digitonin solution. NOTE: Use only a fresh solution of digitonin.

CAUTION: Digitonin is toxic! Use a face mask, gloves and a lab coat.

6. Add PBS with proteinase inhibitor (prepared in step 2.4) to the final volume of $1.5 \mathrm{~mL}$. Centrifuge at $10,000 \times \mathrm{g}$ for $10 \mathrm{~min}$ at $+4{ }^{\circ} \mathrm{C}$. Remove the supernatant. In this step, mitochondria are pelleted.

7. Resuspend the mitochondrial pellet in mitochondrial buffer. The volume of mitochondrial buffer is half of the volume of PBS in step 2.4 .

8. Prepare fresh $10 \%$ lauryl maltoside in PBS containing protease inhibitor (prepared in step 2.4 ). $1 \mathrm{~mL}$ is sufficient for 10 samples. Add $10 \%$ lauryl maltoside to the final concentration of $1 \%$. Incubate on ice for $15 \mathrm{~min}$ (this step can be longer up to a couple of hours).

9. Centrifuge at $20,000 \times g$ for $20 \mathrm{~min}$ at $+4{ }^{\circ} \mathrm{C}$. Collect the supernatant into a new tube. Measure the protein concentration by the Bradford method using a kit. Add sample buffer, a volume that is half of the volume of lauryl maltoside used in step 2.8. Samples can be stored at -80 ${ }^{\circ} \mathrm{C}$ for up to 6 months. 


\title{
3. Preparation of gradient gel for BN-PAGE
}

1. Pour the gradient gel for BN-PAGE at room temperature. Place the gradient maker on a stir plate and connect it with flexible tubing to the peristaltic pump. Attach an infusion set with the needle to the tubing. Place a magnetic stirrer into the proximal chamber of the gradient maker. Wash the tubing with $\mathrm{dH}_{2} \mathrm{O}$ at maximum pump speed for 10 min.

2. Empty the tubing and the gradient maker. Use a pipet to remove any leftover $\mathrm{dH}_{2} \mathrm{O}$ in the channel between chambers of the gradient maker. Close the channel and tubing with the valve.

3. Using casting frame and clamps assemble two glass plates in the holder, which has a hole on the bottom, and place it on the stand. Make sure that it is more or less on a straight surface with a water balance. Place the needle connected to the tubing between the glass plates.

4. Prepare $6 \%$ and $15 \%$ gel solutions (Table 2). Keep on ice. Add ammonium persulphate (APS) and tetramethylenediamine (TEMED) (they start polymerization) last. Mix gently to avoid making air bubbles.

5. Load the proximal end of the gradient-gel-mixer tubing chamber with $6 \%$ gel and the distal end with $15 \%$ gel. The total volume of the gel should be equal to the volume of the separating gel between the glass plates. Thus, use $2.6 \mathrm{~mL}$ of $6 \%$ gel and $2.1 \mathrm{~mL}$ of $15 \%$ gel to the gradient gel mixer for one $8.3 \mathrm{~cm} \times 7.3 \mathrm{~cm}$ sized gel.

6. Switch on the magnetic stirrer, and open the tubing and channel between chambers of the gradient maker. Immediately switch on the peristaltic pump to $5 \mathrm{~mL} / \mathrm{min}$. Fill the glass plates, and do not allow bubbles to enter the gel. Remove the needle when there is no gel in the tubing.

7. Gently overlay the gel with $\mathrm{dH}_{2} \mathrm{O}$. Keep the gel at room temperature for at least $1 \mathrm{~h}$ for polymerization.

8. Immediately after pouring the gel, wash the tubing by filling the gradient chambers with $\mathrm{dH}_{2} \mathrm{O}$ and using the peristaltic pump at maximal speed. When preparing two and more gels, always clean the system in between.

9. Prepare $1 x$ gel buffer by mixing $3 \mathrm{~mL}$ of $3 x$ gel buffer and add $6 \mathrm{~mL}$ of $\mathrm{dH}_{2} \mathrm{O}$. Remove $\mathrm{dH}_{2} \mathrm{O}$ from the surface of the gel with filter paper gently. Wash the surface of the gel with $1 \times$ gel buffer. Remove $1 \times$ gel buffer with filter paper gently.

1. Avoid fibers from the filter paper on the gel. To ensure this, cut the paper to small pieces with scissors, but do not tear the paper.

10. Place the comb between the glass plates, but do not immerse it fully to be able to pour the stacking gel under the comb. Prepare the $4 \%$ stacking gel (Table 2). Add APS and TEMED last as they start polymerization easily. Mix gently avoiding air bubbles.

11. Overlay the stacking gel, avoiding bubbles under the comb, and immerse the comb fully. Let the stacking gel polymerize for at least 30 min. Remove the comb and wash the wells with $1 x$ gel buffer using a pipet.

1. After polymerization, the gel can be stored at $+4{ }^{\circ} \mathrm{C}$ for a couple of days. To store the gel, wrap the gel in paper soaked with $\mathrm{dH}_{2} \mathrm{O}$ and plastic film to prevent drying of the gel.

\section{Blue native gel electrophoresis}

\author{
NOTE: To prevent degradation of OXPHOS complexes run electrophoresis at $+4{ }^{\circ} \mathrm{C}$. Use pre-chilled buffers.
}

1. Load the gel cassette with the blue cathode buffer until the bottom of the wells. Loading of the samples is easier when the cassette is not filled to the top. Wash the wells with the blue cathode buffer and then fill the wells with the blue cathode buffer using pipet.

2. Load the samples (5-30 $\mu \mathrm{g}$ of protein) into wells. Gently fill the gel cassette to the top with the blue cathode buffer and the tank with anode buffer.

3. Run the gel for $15 \mathrm{~min}$ at a constant voltage of $40 \mathrm{~V}$. Then increase the voltage to $80 \mathrm{~V}$ (or use a constant current of $6 \mathrm{~mA}$ ), do not exceed 10 $\mathrm{mA}$. Run the gel until the dye reaches $2 / 3$ of the gel length.

4. Replace the blue cathode buffer with cathode buffer and continue electrophoresis until the dye front has run out. In total, electrophoresis takes about 3 hours.

5. Retrieve the glass plates and transfer the proteins to the polyvinylidene fluoride (PVDF) membrane by semi-dry blotting. Use a constant voltage of $25 \mathrm{~V}$ and a current limited to $1.0 \mathrm{~A}$ for $30 \mathrm{~min}$.

1. Alternatively, use wet blotting to transfer the OXPHOS complexes to a PVDF membrane.

6. Continue with blocking of the membrane and antibody incubation according to the standard immunoblotting protocol. Use the primary antibodies against subunits of OXPHOS complexes sequentially.

NOTE: For example, use anti-NDUFA9 antibody (1:2000) for complex I, anti-SDHA antibody (1:2000) for complex II, anti-UQCRC2 antibody (1:1000) for complex III, anti-COX1 antibody (1:2000) for complex IV and anti-ATP5A antibody (1:2000) for complex V. The list of the antibodies is presented in the Table of Materials.

\section{Representative Results}

Using BN-PAGE, defects in the assembly of mitochondrial OXPHOS complexes can be investigated. Figure 1A shows the assembly of respiratory chain complexes in human neuroblastoma cells (SH-SY5Y) treated with chloramphenicol, which specifically inhibits translation of mtDNA-encoded OXPHOS subunits. Chloramphenicol depleted the OXPHOS complexes that contained mitochondrially-encoded subunits (complex I, III, IV; Figure 1A), while complex II, which is exclusively encoded by nuclear genes, was compensatorily upregulated. Complex V was not immunoblotted here.

Multiple freeze-thaw cycles destroy OXPHOS complexes. Figure 1B demonstrates the degradation of OXPHOS complexes by the freezethaw cycles. Mitochondrial respiratory complexes in sample 3 that have undergone multiple freeze-thaw cycles have a lower band intensity and are shifted in comparison to the same samples, which were frozen only once. An uncropped western blot image of Figure 1B is shown in Supplementary Figure 1. 
The quality of the gel is important for sharp and clear bands. Figure 1C shows an immunoblot from a gel that did not polymerize well. Stripping of the membrane also can affect the detection of OXPHOS complexes. In Figure 1D, complex I was detected before or after stripping. The signal to noise ratio is much lower after stripping.

A
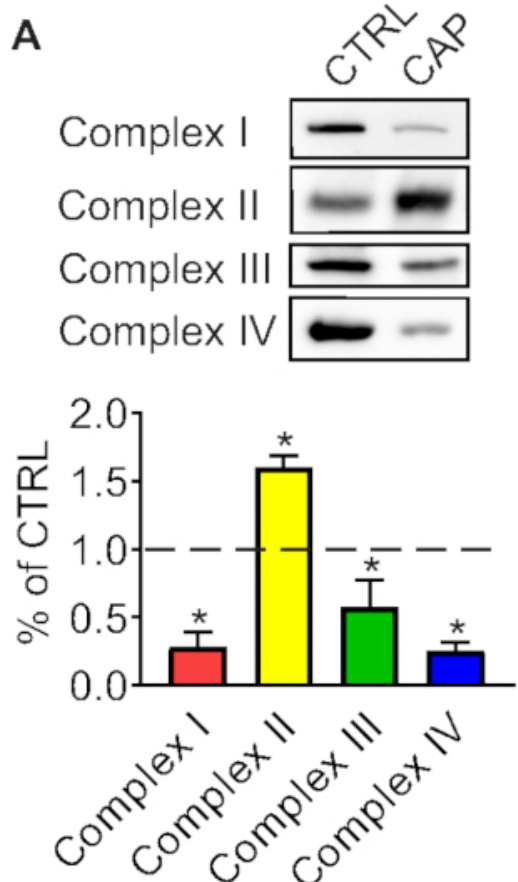

B

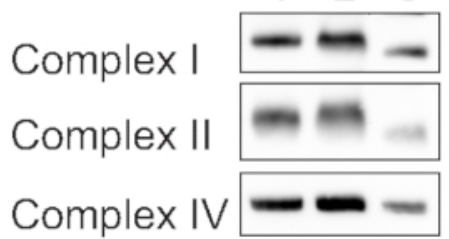

C

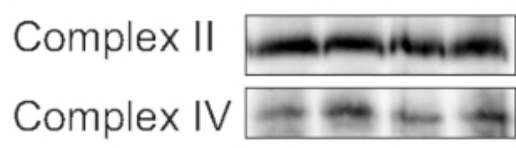

D Complex I

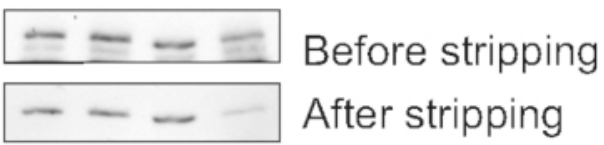

Figure 1. BN-PAGE immunoblots from successful and sub-optimal experiments. (A) Depletion of OXPHOS complexes by inhibitor of mitochondrial translation. Human neuroblastoma cells (SH-SY5Y) were treated with chloramphenicol (CAP) for 48 hours. CTRL, control cells without chloramphenicol treatment. The lower panel shows the quantification of the immunoblot. The value of the control is taken as 1 (shown as a dashed line). Data are presented as mean $\pm S D, n=3,{ }^{*} P<0.0001$ as compared to control using unpaired Student's t-tests. (B) Disruption of OXPHOS complexes by multiple freeze-thaw cycles. Samples 1-3 were prepared from human osteosarcoma cells (143B) in the same conditions. Samples number 1 and 2 were frozen and melted only once, while the sample number 3 underwent four freeze-thaw cycles. (C) BN-PAGE immunoblots of OXPHOS complexes isolated from HEK293 cells. The smearing of the bands is caused by low quality of the gel. (D) Effect of stripping on the detection of OXPHOS complexes. Detection of Complex I in human neuroblastoma cells (SH-SY5Y) before and after stripping of the same membrane. OXPHOS complexes were detected using anti-NDUFA9 antibody (complex I), anti-SDHA antibody (complex II), antiUQCRC2 antibody (complex III) and anti-COX1 antibody (complex IV). Please click here to view a larger version of this figure. 


\begin{tabular}{|c|c|c|}
\hline Buffer or solution & Composition & Recipe \\
\hline \multirow[t]{4}{*}{$3 \times$ gel buffer } & 1.5 M aminocaproic acid & $3.94 \mathrm{~g}$ of aminocaproic acid \\
\hline & 150 mM Bis-tris & $0.63 \mathrm{~g}$ of Bis-tris \\
\hline & $\mathrm{dH}_{2} \mathrm{O}$ & Add $\mathrm{dH}_{2} \mathrm{O}$ to $20 \mathrm{~mL}$ \\
\hline & $\mathrm{pH} 7.0$ & Adjust $\mathrm{pH}$ to 7.0 \\
\hline \multirow[t]{4}{*}{ Cathode buffer } & $15 \mathrm{mM}$ Bis-tris & $3.14 \mathrm{~g}$ of Bis-tris \\
\hline & $50 \mathrm{mM}$ tricine & $8.96 \mathrm{~g}$ of tricine \\
\hline & $\mathrm{dH}_{2} \mathrm{O}$ & Add $\mathrm{dH}_{2} \mathrm{O}$ to $1000 \mathrm{~mL}$ \\
\hline & $\mathrm{pH} 7.0$ & Adjust $\mathrm{pH}$ to 7.0 \\
\hline \multirow[t]{5}{*}{ Blue cathode buffer } & $0.02 \%$ coomassie blue $\mathrm{G}$ & $0.04 \mathrm{~g}$ of Serva blue $\mathrm{G}$ \\
\hline & $15 \mathrm{mM}$ Bis-tris & \multirow[t]{4}{*}{$200 \mathrm{~mL}$ of Cathode buffer } \\
\hline & $50 \mathrm{mM}$ tricine & \\
\hline & $\mathrm{dH}_{2} \mathrm{O}$ & \\
\hline & $\mathrm{pH} 7.0$ & \\
\hline \multirow[t]{3}{*}{ Anode buffer } & $50 \mathrm{mM}$ Bis-tris & $20.93 \mathrm{~g}$ \\
\hline & $\mathrm{dH}_{2} \mathrm{O}$ & Add $\mathrm{dH}_{2} \mathrm{O}$ to $2000 \mathrm{~mL}$ \\
\hline & $\mathrm{pH} 7.0$ & Adjust $\mathrm{pH}$ to 7.0 \\
\hline \multirow[t]{5}{*}{ Mitochondrial buffer } & 1.75 M aminocaproic acid & $0.5 \mathrm{~mL}$ of $3 \times$ gel buffer \\
\hline & $75 \mathrm{mM}$ Bis-tris & $0.5 \mathrm{~mL}$ of $2 \mathrm{M}$ aminocaproic acid \\
\hline & $2 \mathrm{mM}$ EDTA & $4 \mu \mathrm{L}$ of $500 \mathrm{mM}$ EDTA \\
\hline & $\mathrm{dH}_{2} \mathrm{O}$ & - \\
\hline & $\mathrm{pH} 7.0$ & - \\
\hline \multirow[t]{3}{*}{ Sample buffer } & $750 \mathrm{mM}$ aminocaproic acid & $0.94 \mathrm{~mL}$ of $2 \mathrm{M}$ aminocaproic acid \\
\hline & $5 \%$ comassie blue $\mathrm{G}$ & $0.125 \mathrm{~g}$ of Serva blue $\mathrm{G}$ \\
\hline & $\mathrm{dH}_{2} \mathrm{O}$ & Add $\mathrm{dH}_{2} \mathrm{O}$ to $2.5 \mathrm{~mL}$ \\
\hline \multirow[t]{2}{*}{ 2M aminocaproic acid } & $2 \mathrm{M}$ aminocaproic acid & $2.62 \mathrm{~g}$ of aminocaproic acid \\
\hline & $\mathrm{dH}_{2} \mathrm{O}$ & Add $\mathrm{dH}_{2} \mathrm{O}$ to $10 \mathrm{~mL}$ \\
\hline
\end{tabular}

Table 1. Buffers and solutions used for BN-PAGE.

\begin{tabular}{|l|l|l|l|}
\hline Blue native gels & Separating 6\% gel & Separating $15 \%$ gel & Stacking $4 \%$ gel \\
\hline $3 \times$ gel buffer & $3.3 \mathrm{~mL}$ & $3.3 \mathrm{~mL}$ & $1.64 \mathrm{~mL}$ \\
\hline $40 \%$ Acrylamide/Bis $37.5: 1$ & $1.5 \mathrm{~mL}$ & $3.75 \mathrm{~mL}$ & $0.5 \mathrm{~mL}$ \\
\hline $\mathrm{dH}_{2} \mathrm{O}$ & $5.14 \mathrm{~mL}$ & $0.93 \mathrm{~mL}$ & $2.77 \mathrm{~mL}$ \\
\hline Glycerol & 0 & $2 \mathrm{~mL}$ & 0 \\
\hline $10 \%$ Ammonium persulfate & $60 \mu \mathrm{L}$ & $10 \mu \mathrm{L}$ & $60 \mu \mathrm{L}$ \\
\hline TEMED & $4 \mu \mathrm{L}$ & $6 \mu \mathrm{L}$ \\
\hline Total volume & $10 \mathrm{~mL}$ & $2 \mu \mathrm{LL}$ \\
\hline${ }^{*}$ Make always fresh $10 \%$ ammonium persulfate in $\mathrm{dH}_{2} \mathrm{O}$ & $10 \mathrm{~mL}$ & $5 \mathrm{~mL}$ \\
\hline
\end{tabular}

Table 2. Recipes of gel for BN-PAGE.

Supplementary Figure 1. Uncropped western blot image of Figure 1B. Please click here to download this figure.

\section{Discussion}

One of the most critical parts of the protocol is to preserve intact OXPHOS complexes during sample preparation, storage and gel electrophoresis. Thus, mitochondria should be isolated at $+4{ }^{\circ} \mathrm{C}$ and the samples should not undergo freeze-thaw cycles. OXPHOS complexes can only tolerate one freeze-thaw cycle during the whole procedure. Multiple freeze-thaw cycles destroy OXPHOS complexes (Figure 1B).

Control and experimental samples that are to be compared should be prepared in parallel to avoid any differences in storage conditions, which 
might give misleading results. If it is not possible to perform all the steps of the protocol in parallel with all the samples, we recommend to freeze washed cell pellets at $-80^{\circ} \mathrm{C}$ (step 1.4 in this protocol) and later carry out the rest of the protocol for all the samples together at least until gel electrophoresis. Special attention should be paid to the cleanliness of the electrophoresis apparatus (tank, cassette). If the same apparatus is used for SDS-PAGE, wash it very well before BN-PAGE. Any residual SDS can cause dissociation of the OXPHOS complexes during electrophoresis.

High-quality gradient gels are another critical part of the protocol. Precast gels for blue native PAGE are commercially available; however, it is not recommended to use them, since the buffers used for commercial gels might have a composition, which is different from the sample buffers. The gradient of the gel used here (6-15\%) is optimal for separation of individual OXPHOS complexes. To detect higher-order supramolecular structures of OXPHOS, also known as respiratory chain supercomplexes ${ }^{14}$, some optimization is required ${ }^{11}$.

For the visualization of OXPHOS complexes use the specific antibodies sequentially, based on their properties. For example, use first the antibody that gives the weakest signal and the antibody with the strongest signal last. This is important since the stripping weakens the detection (Figure 1D). The composition of respiratory chain complexes can be investigated if after BN-PAGE the gel is subjected to the second dimension SDS-PAGE ${ }^{15}$.

The protocol described here suggests using antibodies against individual OXPHOS complexes sequentially. However, the commercially available OXPHOS antibody cocktail can be used to detect all five OXPHOS complexes simultaneously. Nevertheless, to be able to detect incompletely assembled OXPHOS complexes and define their identity, antibodies against individual OXPHOS complexes should be used sequentially. This step is time-consuming; however, it can be essential for testing new experimental conditions and models.

The concentration of digitonin used for mitochondrial isolation should be optimized for the specific cell type. As a detergent, digitonin permeabilizes cell membranes. The optimal concentration of digitonin efficiently permeabilizes the plasma membrane of the cells leaving the mitochondrial membranes intact. Too low concentration of digitonin causes high contamination of the mitochondrial extracts while too high concentration damages the mitochondrial membranes and reduces the total mitochondrial yield. The optimal digitonin/protein ratio $(\mathrm{g} / \mathrm{g})$ varies from 0.3 to 1 . Western blot analysis of proteins extracted from pellets and supernatants can be used to test the optimal concentration of digitonin ${ }^{16}$.

This protocol does not include protein loading control on the immunoblot; therefore, the protein concentration of extracted complexes should be carefully measured at least in triplicates to ensure equal loading. In addition, the samples can be run in BN-PAGE in replicates. If the assembly of selective OXPHOS complexes is impaired, unaffected complexes can serve as loading controls.

The estimation of the molecular mass of the protein complexes in the BN-PAGE is challenging ${ }^{18}$. The current protocol does not include the molecular weight marker; therefore, to estimate the assembly of the OXPHOS complexes, the control samples containing unaffected complexes should always be included in the analysis. The control samples for BN-PAGE are usually untreated or wild type cells.

The method presented here is optimized for the detection of mitochondrial respiratory chain complexes; however, it can also be applied for the evaluation of oligomerization of mitochondrial proteins ${ }^{19}$. In addition, the assembly rate of OXPHOS complexes can be studied by first depleting mitochondrial-encoded subunit containing complexes by chloramphenicol and then following their recovery after removal of chloramphenicol ${ }^{10}$. Thus, BN-PAGE can be used to evaluate steady-state levels and assembly of OXPHOS for different applications including molecular diagnostics of human mitochondrial disorders ${ }^{9,11}$.

\section{Disclosures}

No conflicts of interest declared.

\section{Acknowledgments}

Helsinki University Library is thanked for the financial support in publishing.

\section{References}

1. Osellame, L.D., Blacker, T.S., Duchen, M.R. Cellular and molecular mechanisms of mitochondrial function. Best Practice, Research. Clinical Endocrinology \& Metabolism. 26 (6), 711-723 (2012).

2. Jang, J.Y., Blum, A., Liu, J., Finkel, T. The role of mitochondria in aging. The Journal of Clinical Investigation. 128 (9), $3662-3670$ (2018).

3. Nunnari, J., Suomalainen, A. Mitochondria: in sickness and in health. Cell. 148 (6), 1145-1159 (2012).

4. Cogliati, S., Lorenzi, I., Rigoni, G., Caicci, F., Soriano, M.E. Regulation of Mitochondrial Electron Transport Chain Assembly. Journal of Molecular Biology. (2018).

5. Fernandez-Vizarra, E., Tiranti, V., Zeviani, M. Assembly of the oxidative phosphorylation system in humans: what we have learned by studying its defects. Biochimica et Biophysica Acta. 1793 (1), 200-211 (2009).

6. Signes, A., Fernandez-Vizarra, E. Assembly of mammalian oxidative phosphorylation complexes I-V and supercomplexes. Essays in Biochemistry. 62 (3), 255-270 (2018).

7. Schagger, H., von Jagow, G. Blue native electrophoresis for isolation of membrane protein complexes in enzymatically active form. Analytical Biochemistry. 199 (2), 223-231 (1991).

8. Wittig, I., Braun, H.P., Schagger, H. Blue native PAGE. Nature Protocols. 1 (1), 418-428 (2006).

9. Gotz, A. et al. Exome sequencing identifies mitochondrial alanyl-tRNA synthetase mutations in infantile mitochondrial cardiomyopathy. American Journal of Human Genetics. 88 (5), 635-642 (2011).

10. Wittig, I., Schagger, H. Advantages and limitations of clear-native PAGE. Proteomics. 5 (17), 4338-4346 (2005). 
11. Luo, X., Wu, J., Jin, Z., Yan, L.J. Non-Gradient Blue Native Polyacrylamide Gel Electrophoresis. Current Protocols in Protein Science. 87, 19.29.1-19.29.12 (2017).

12. Jha, P., Wang, X., Auwerx, J. Analysis of Mitochondrial Respiratory Chain Supercomplexes Using Blue Native Polyacrylamide Gel Electrophoresis (BN-PAGE). Current Protocols in Mouse Biology. 6 (1), 1-14 (2016).

13. Preston, C.C. et al. Aging-induced alterations in gene transcripts and functional activity of mitochondrial oxidative phosphorylation complexes in the heart. Mechanisms of Ageing and Development. 129 (6), 304-312 (2008).

14. Hilander, T., Konovalova, S., Terzioglu, M., Tyynismaa, H. Analysis of Mitochondrial Protein Synthesis: De Novo Translation, Steady-State Levels, and Assembled OXPHOS Complexes. Current Protocols In Toxicology., e56 (2018).

15. Lobo-Jarne, T., Ugalde, C. Respiratory chain supercomplexes: Structures, function and biogenesis. Seminars in Cell \& Developmental Biology. 76, 179-190 (2018).

16. Fiala, G.J., Schamel, W.W., Blumenthal, B. Blue native polyacrylamide gel electrophoresis (BN-PAGE) for analysis of multiprotein complexes from cellular lysates. Journal of Visualized Experiments. (48), 10.3791/2164 (2011).

17. Itahana, K., Clegg, H.V., Zhang, Y. ARF in the mitochondria: the last frontier? Cell Cycle (Georgetown, Tex.). 7 (23), $3641-3646$ (2008).

18. Wittig, I., Beckhaus, T., Wumaier, Z., Karas, M., Schagger, H. Mass estimation of native proteins by blue native electrophoresis: principles and practical hints. Molecular \& Cellular Proteomics. 9 (10), 2149-2161 (2010).

19. Konovalova, S. et al. Redox regulation of GRPEL2 nucleotide exchange factor for mitochondrial HSP70 chaperone. Redox Biology. 19, $37-45$ (2018).

20. Konovalova, S. et al. Exposure to arginine analog canavanine induces aberrant mitochondrial translation products, mitoribosome stalling, and instability of the mitochondrial proteome. The International Journal of Biochemistry \& Cell Biology. 65, 268-274 (2015).

21. Ahmed, S.T. et al. Using a quantitative quadruple immunofluorescent assay to diagnose isolated mitochondrial Complex I deficiency. Scientific Reports. 7 (1), 15676-017-14623-2 (2017). 